\title{
Caracterización de los Componentes Óseos de la Articulación Temporomandibular. Una Revisión de la Literatura
}

\author{
Characterization of Bone Components of the Temporomandibular Joint. A Literature Review.
}

Fuentes, R. ${ }^{* * * *}$; Cantín, M. ${ }^{* * *, * * * *, * * * * * *}$; Ottone, N. E. ${ }^{* *, * * * *}$ \& Bucchi, C.,**

FUENTES, R.; CANTÍN, M.; OTTONE, N. E. \& BUCCHI, C. Caracterización de los componentes óseos de la articulación temporomandibular. Una revisión de la literatura. Int. J. Morphol., 33(4):1569-1576, 2015.

RESUMEN: La articulación temporomandibular (ATM) es una articulación sinovial bilateral de tipo ginglimo-artroidal que anatómicamente está conformada por el cóndilo mandibular, fosa mandibular y tubérculo articular. Es necesario tener un acabado conocimiento de la anatomía de la ATM para una correcta comprensión de su funcionamiento. La descripción anatómica de las estructuras óseas presente en la literatura científica, y que componen la ATM, no es del todo exacta, con diversas diferencias sobre la delimitación del cóndilo mandibular, las fisuras presentes en la fosa mandibular y las superficies articulares de la fosa mandibular y tubérculo articular, así como una amplia variabilidad de términos para referirse a cada estructura anatómica. Se realizó una revisión sistemática de textos y artículos científicos sobre la descripción anatómica de los componentes óseos de la ATM y los términos para referirse a ellos, comparándolos con los términos establecidos por la Terminología Anatómica Internacional.

PALABRAS CLAVE: Articulación temporomandibular; Cóndilo mandíbular; Tubérculo articular; Fosa mandibular; Terminologia Anatomica Internacional.

\section{INTRODUCCIÓN}

La articulación temporomandibular (ATM) es una articulación sinovial bilateral de tipo ginglimo-artroidal, que permite la rotación y desplazamiento del cóndilo mandibular (Rubiano, 2005; Ramfjord \& Ash, 1972), y movimientos de apertura, cierre, protrusión, retrusión y lateralidad de la mandíbula (Latarjet \& Ruiz Liard, 2005). Anatómicamente, está conformada por el cóndilo mandibular, fosa mandibular y tubérculo articular, pertenecientes al hueso temporal (Velayos \& Santana, 2001; Vasconcellos et al., 2007).

En los textos de anatomía existe discrepancia al describir la delimitación de las estructuras óseas que componen la ATM. Así, no hay una descripción unificada sobre cuál es el límite posterior de la porción articular de la fosa mandibular; algunos autores la definen en la fisura timpanoescamosa (Alves \& Candido, 2009), fisura petrotimpánica (Sosa, 2006; Figún \& Garino, 2002; Velayos \& Santana) y otros en la Cisura de Glasser (sic) (Martínez Ross, 1992), lo que evidencia una falta de coincidencia con respecto a la posición y terminología adecuada de las fisuras presentes en la fosa mandibular. Así mismo, el término proceso condilar, que involucra al cuello de la mandíbula y al cóndilo mandibular (Figún \& Garino; Comité Federal sobre Terminología Anatómica, 2001), es utilizado como sinónimo del termino cóndilo mandibular (Velayos \& Santana; Okeson, 2003; Sicher \& Dubrul, 1991).

La Terminologia Anatomica Internacional (TAI) establece un listado de términos unificados, descriptivos y carentes de epónimos, con el objetivo de facilitar la comunicación entre la comunidad científica. Sin embargo, no es extraño encontrar literatura especializada (especialmente libros), que continúa utilizando términos distintos a los establecidos en TAI o varios términos para una misma estructura anatómica simultáneamente. Además, existen estructuras anatómicas relacionadas a la ATM que no están mencionadas en TAI (Fuentes et al., 2014a), como el ligamento discomaleolar (Fuentes et al., 2014b).

* Programa Magister en Odontología, Facultad de Odontología, Universidad de La Frontera, Temuco, Chile.

** Centro de Investigación en Ciencias Odontológicas CICO, Facultad de Odontología, Universidad de La Frontera, Temuco, Chile.

*** Programa de Doctorado en Ciencias Morfológicas, Universidad de La Frontera, Temuco, Chile.

***** Centro de investigación CIMA. Facultad de Odontología, Universidad de La Frontera, Temuco, Chile.

******* Centro de Investigación en Ciencias Biomédicas, Universidad Autónoma de Chile, Temuco, Chile. 
El objetivo de esta investigación fue revisar la literatura especializada para entregar una descripción anatómica del cóndilo mandibular, tubérculo articular y fosa mandibular, utilizando la evidencia científica disponible y nombrándolos según lo establecido por Terminologia Anatomica Internacional.

\section{MATERIAL Y MÉTODO}

Revisión de la literatura especializada, que consideró libros de anatomía y artículos científicos. Se realizó la búsqueda de libros de anatomía general o específica que trataban sobre la ATM, escritos en idiomas español, portugués, alemán e inglés, publicados entre los años 1972 y 2013. Se buscaron de manera específica los términos referentes a los componentes óseos de la ATM (cóndilo, fosa mandibular y tubérculo articular).

En cuanto a los artículos científicos, se realizó una búsqueda sistemática en las bases de datos MEDLINE, SciELO, Scopus y Cochrane. El límite temporal para la búsqueda se estableció desde la fecha inicial permitida por cada base, hasta el 30 Diciembre del año 2014, en todos los casos. Los términos utilizados para la búsqueda fueron: ("mandibular condyle"[All Fields] OR "mandibular condyle"[MeSH Terms]) OR condyle[All Fields]) AND "mandibular fossa"[All Fields]) OR "glenoid fossa"[All Fields]) OR "articular fossa"[All Fields]) AND "articular tubercle"[All Fields]) OR "articular eminence"[All Fields] AND ("anatomy and histology"[Subheading] OR ("anatomy"[All Fields] AND "histology"[All Fields]) OR "anatomy and histology"[All Fields] OR "morphology"[All Fields]) AND "humans"[MeSH Terms]. Se utilizaron los mismos términos para ingresar al buscador de la base SciELO.
Criterios de inclusión y exclusión. Se incluyeron artículos científicos referentes a estudios de tipo observacional descriptivo, realizados en cráneos humanos adultos (18 o más años), sin alteraciones o patologías, orientados a la descripción morfológica o morfométrica de los componentes óseos de la ATM (cóndilo, fosa mandibular y tubérculo articular). En caso de que los estudios realizaran mediciones, éstas debían estar medidas directamente en los cráneos y no mediante imagenología. Los artículos estuvieron con disponibilidad de texto completo, escritos en idioma español, inglés y portugués. No se aplicó restricción por tamaño de la muestra. Fueron excluidos cartas al editor, revisiones bibliográficas y artículos técnicos.

Metodología de revisión. La revisión de artículos se realizó en dos etapas por un investigador. Una primera revisión se llevó a cabo leyendo los títulos o resúmenes de los artículos inicialmente entregados por la búsqueda electrónica, para estudiar su atingencia al tema. De los artículos seleccionados, se leyó el texto completo y verificó su cumplimiento con los criterios de inclusión. Adicionalmente, se realizó una búsqueda manual de los artículos referenciados en la literatura incluida en esta revisión.

Extracción de datos. La extracción de los datos fue realizada por un investigador. Se consignó la descripción morfológica o morfometría de las estructuras óseas de estudio (cóndilo mandibular, fosa mandibular y tubérculo articular), así como términos empleados para referirse dichas estructuras. La información recopilada fue ingresada a una ficha de registro diseñada previamente. La descripción anatómica fue clasificada según estructura, y los términos encontrados se agruparon y compararon entre sí y con la TAI, en su versión latín, inglés y español (2001).

Tabla I. Comparación de los términos utilizados en la literatura y los establecidos por la Terminologia Anatomica Internacional (TAI) para referirse al componente mandibular de la ATM.

\begin{tabular}{|c|c|c|}
\hline Términos TAI & $\begin{array}{l}\text { Términos utilizados en } \\
\text { la literatura científica }\end{array}$ & Referencias bilbiográficas \\
\hline \multirow[t]{2}{*}{ Pro ceso condilar (Processus condylaris) } & Apófisis condilar & Latarjet \& Ruiz Liard (2005) \\
\hline & Proceso condilar & Alves \& Candido (2009) \\
\hline \multirow{6}{*}{$\begin{array}{l}\text { Cóndilo de la mandíbula o C abeza de la } \\
\text { mandíbula (Condylus mandibulae, Caput } \\
\text { mandibulae) }\end{array}$} & Cabeza de la mandíbula & $\begin{array}{l}\text { Pertes \& Gross (2005); Sicher \& Dubrul (1991); Schunke } \\
\text { et al. (2010) }\end{array}$ \\
\hline & Cabeza articular & Schunke et al. (2010) \\
\hline & Proceso condilar & Okeson (2003); Sicher \& Dubrul (1991) \\
\hline & Processus condylaris & Velayos \& Santana (2001) \\
\hline & Cóndilo del maxilar & Ramfjord \& Ash (1972); Martinez Ross (1992) \\
\hline & Cóndilo de la mandíbula & $\begin{array}{l}\text { Latarjet \& Ruiz Liard (2005); Velayos \& Santana (2001); } \\
\text { Figún \& Garino (2002); Rubiano (2005); Okeson (2003) }\end{array}$ \\
\hline $\begin{array}{l}\text { Cuello de la mandíbula (Collum } \\
\text { mandibulae) }\end{array}$ & $\begin{array}{l}\text { Cuello mandibular } \\
\text { Cuello del cóndilo }\end{array}$ & $\begin{array}{l}\text { Sicher \& Dubrul (1991); Latarjet \& Ruiz Liard (2005) } \\
\text { Rubiano (2005) }\end{array}$ \\
\hline
\end{tabular}




\section{RESULTADOS}

Un total de 16 libros fueron analizados, cinco correspondientes a textos de anatomía general, cinco a anatomía aplicada y seis a especialidades odontológicas; once libros fueron publicados en idioma español, dos en portugués, dos en alemán y uno en inglés.

La estrategia de búsqueda en las bases de datos, encontró 838 artículos referentes al tema, pero solo cuatro cumplieron los criterios de selección. Se incluyó un artículo más luego de la búsqueda manual. En la Figura 1 se muestra el flujograma de revisión y selección de los 5 artículos.

En la Tabla I, se muestran los términos encontrados en la literatura científica para referirse al proceso condilar, cóndilo de la mandíbula y cuello de la mandíbula. En la Tabla II se observan los términos encontrados en la literatura científica para referirse a la fosa mandibular y tubérculo articular. En ambas tablas se exponen los términos establecidos por la TAI.

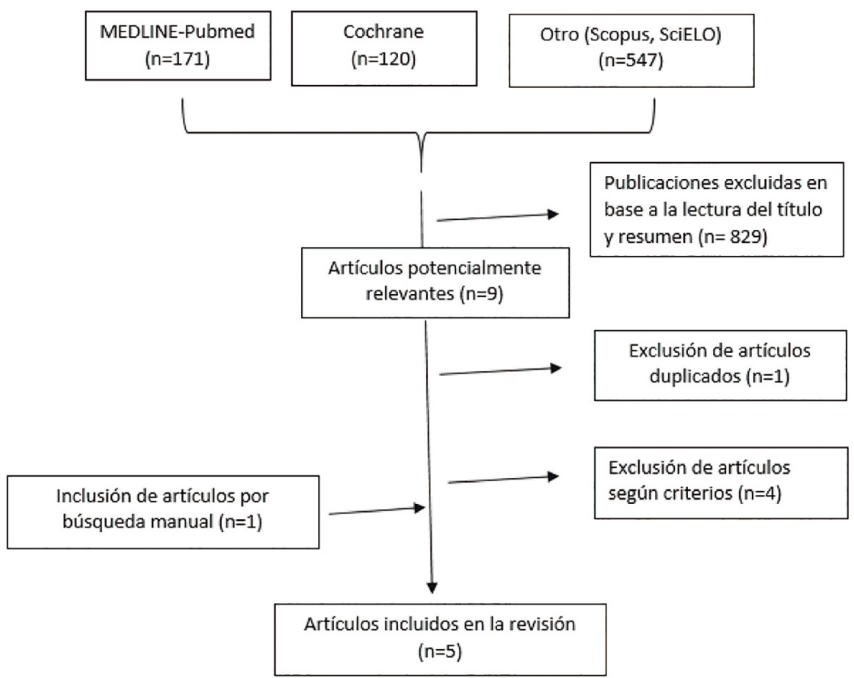

Fig. 1. Flujograma de los artículos incluidos obtenidos por la búsqueda electrónica.

\section{DISCUSIÓN}

Proceso condilar (Processus condylaris). El proceso condilar comprende al cuello y cóndilo de la mandíbula (o cabeza de la mandíbula) (Figún \& Garino; Comité Federal sobre Terminología Anatómica). Sin embargo, algunos autores utilizan el término proceso condilar como sinónimo del término cóndilo de la mandíbula (Velayos \& Santana; Okeson; Sicher \& Dubrul) (Tabla I).

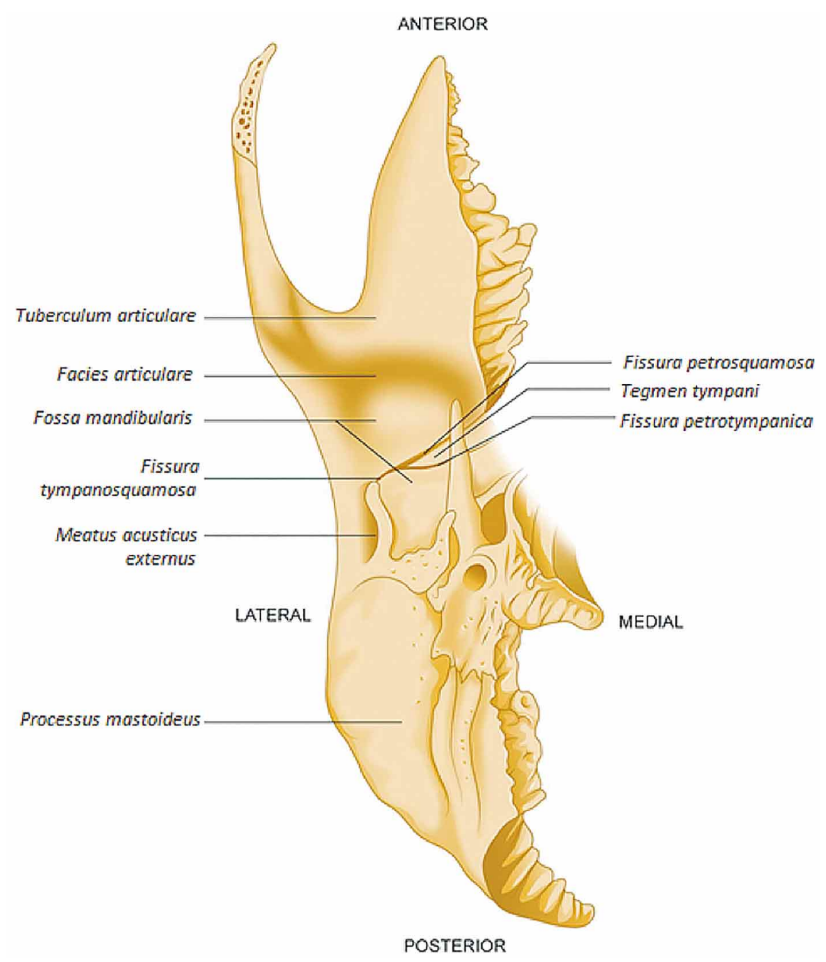

Fig. 2. Vista inferior de la fosa mandibular derecha. Se puede observar la distribución de las tres fisuras presentes en la fosa mandibular: la fisura timpanoescamosa se bifurca en la fisura petroescamosa por mesial y petrotimpanica por distal.

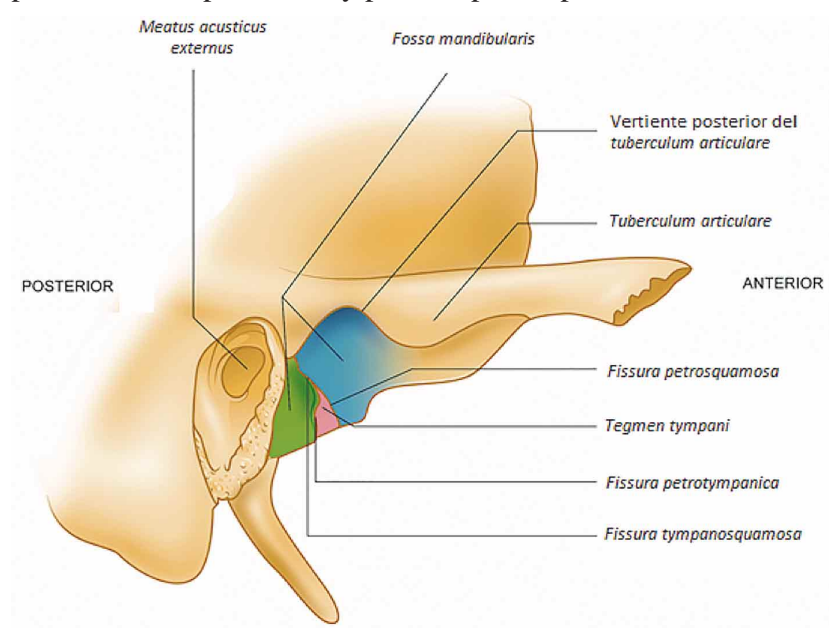

Fig. 3. Vista lateral de la fosa mandibular derecha. Color azul: porción escamosa del hueso temporal, color verde: porción timpánica del hueso temporal, color rosado: porción petrosa del hueso temporal. Sólo la porción de color azul es una superficie articular.

El cóndilo de la mandíbula o cabeza de la mandíbula (Condylus mandibulae, Caput mandibulae). Se define como una eminencia (Velayos \& Santana; Figún \& Garino), cuyo eje mayor se orienta oblicuamente hacia posterior y medial (Figún \& Garino; Martinez Ross), aproximadamente $20^{\circ}$ con respecto al plano coronal. Velayos \& Santana indican que el eje mayor del cóndi- 
lo mandibular guarda la misma dirección que la fosa mandibular.

La literatura revisada concuerda en que es muy convexo en sentido anteroposterior y levemente convexo en sentido latero-medial (Okeson; Bumman \& Lotzmann, 2000; Sicher \& Dubrul; Figún \& Garino). Según Velayos \& Santana, los polos medial y lateral terminan en forma puntiaguda, siendo el polo medial más prominente que el lateral (Okeson; Sicher \& Dubrul). Sin embargo, Sicher \& Dubrul definen que sólo el polo lateral tiene una forma puntiaguda y el polo medial es aplanado. Una línea imaginaria que una ambos polos y que siga la dirección del eje mayor de los cóndilos (hacia posterior y medial) uniéndose en el margen anterior del foramen magno (Foramen magnum) formaría un ángulo de $135^{\circ}$ (Velayos \& Santana) ó entre $150^{\circ}-160^{\circ}$ (Figún \& Garino). En los polos lateral y medial se insertan los ligamentos colaterales lateral y medial, respectivamente (Okeson), uniendo el disco articular con el cóndilo (Sosa). En el polo lateral y en la parte posterior del disco articular se inserta la porción horizontal del ligamento lateral de la ATM (Okeson).

En cuanto a la forma del cóndilo, su descripción es variable. Sosa y Martinez Ross lo describen de forma ovalada, Schunke et al. (2010) de forma cilíndrica y Langton \& Eggleton y Figún \& Garino de forma elíptica. Oberg et al. (1970) en un estudio realizado sobre 102 ATM de cráneos entre 20 a 93 años de edad señalan que desde una vista anterior, la forma más preponderante fue la redondeada o ligeramente convexa (55\%), seguida de una forma aplanada (20\%) o forma de $\mathrm{V}$ invertida u otras formas (25\%), mientras que desde una vista superior se describe una forma oblonga $(44 \%)$, oval o redondeada (36\%) u otras formas (19\%); además, los dichos autores señala que en el adulto existe congruencia entre la forma mediolateral del cóndilo y la del tubérculo articular, así, un cóndilo convexo (desde una vista anterior) era comúnmente correspondido con una forma cóncava del tubérculo, y un cóndilo plano se correspondía con un tubérculo también plano.

Se ha establecido que el cóndilo mandibular posee una dimensión antero-posterior entre 8-10 mm (Velayos \& Santana, Sosa, Okeson; Sicher \& Dubrul) y 9,8 mm (Bumann \& Lotzmann), una dimensión medio-lateral entre 15-20 mm (Velayos \& Santana; Okeson; Sicher \& Dubrul) y 20-22 mm (Figún \& Garino), con medidas específicas para hombres $(21,8 \pm 1,93 \mathrm{~mm})$ y mujeres $(18,7 \pm 1,57)$ (Bumann \& Lotzmann), una dimensión supero-inferior (visto desde anterior) de 10,1 mm en hombres y 9,8 mm en mujeres (Bumann \& Lotzmann). Estas medidas concuerdan con las reportadas por Oberg et al. en 102 ATM, donde la longitud antero-posterior fue de 9,8 $\mathrm{mm}$ y la longitud medio-lateral de $19,8 \mathrm{~mm}$.
Superficie articular (Facies articularis) del cóndilo mandibular. Se describe una cresta roma transversa de aspecto variable (Figún \& Garino) que divide la superficie articular del cóndilo en dos vertientes, una anterior convexa y otra posterior plana, más pequeña. Velayos \& Santana y Alves \& Candido señalan que la vertiente anterior es la verdadera superficie articular, mientras que otros autores describen a la vertiente anterior y al extremo superior de la vertiente posterior como la superficie articular (Figun \& Garino; Sicher \& Dubrul; Langton \& Eggleton; Rubiano). Oberg et $a l$. establecieron que límite medial de la superficie articular se encontraba siempre situada en la porción media del polo medial del cóndilo o bajo ésta, y el limite lateral, en la mayoría de los casos, se encontraba en la parte media del polo lateral y o bajo ésta. Además, los autores concuerdan en que las superficies articulares estarían recubiertas por fibrocartílago (Velayos \& Santana; Langton \& Eggleton; Ramfjord \& Ash; Rubiano) y las no articulares por tejido fibroso (Velayos \& Santana); aunque Martinez-Ross, Figún \& Garino y Alves \& Candido señalan que las superficies articulares están recubiertas por tejido fibroso.

Cuello de la mandíbula (Collum mandibulae). Es la porción de hueso que une el cóndilo de la mandíbula con la rama mandibular (Ramus mandibulae) (Figún \& Garino) y se encuentra levemente inclinado hacia anterior (Figún \& Garino). Da inserción a importantes estructuras ligamentosas y musculares, y alrededor de él se inserta la capsula de la ATM. En la superficie lateral y posterior del cuello se inserta la porción oblicua del ligamento lateral (Ligamentum laterale) de la ATM (Langton \& Eggleton; Okeson). En el tercio superior del cuello, distal a la superficie articular del cóndilo se inserta la porción inferior de la zona bilaminar del disco articular (Discus articularis) (Langton \& Eggleton). Además, el cuello de la mandíbula está provisto de la fosita pterigoidea (Fovea pterigoidea) en su cara anteromedial, la cual da inserción al músculo pterigoideo lateral (Musculi pterygoideus lateralis) (Figún \& Garino; Latarjet \& Ruiz).

Fosa mandibular (Fossa mandibularis). Porción del hueso temporal de forma cóncava (Okeson; Ramfjord \& Ash) en sentido anteroposterior y mediolateral (Velayos \& Santana) y de profundidad variable (Figún \& Garino). Algunos autores (Okeson; Latarjet \& Ruiz; Schunke et al.; Rubiano) señalan que la fosa mandibular está formada exclusivamente por la porción escamosa del hueso temporal, ubicándose anterior a las fisuras presentes en la fosa mandibular (Martinez Ross). Sin embargo, otros señalan que la fosa se extiende desde el segmento anterior del conducto auditivo externo (Figún \& Garino) hasta el tubérculo articular (Latarjet \& Ruiz; Figún \& Garino), comprometiendo así, parte de la porción timpánica y petrosa del hueso temporal (Fig. 2). En sentido transversal, la fosa comprometería des- 
Tabla II. Comparación de los términos utilizados en la literatura y los establecidos por la Terminologia Anatomica Internacional (TAI) para referirse al componente craneal de la ATM.

\begin{tabular}{|c|c|c|}
\hline Términos TAI & $\begin{array}{l}\text { Términos utilizados en } \\
\text { la literatura científica }\end{array}$ & Referencias bilbiográficas \\
\hline \multirow[t]{6}{*}{$\begin{array}{l}\text { Tubérculo articular (Tuberculum } \\
\text { articulare) }\end{array}$} & Tubérculo articular & $\begin{array}{l}\text { Pertes \& Gross (2005), Latarjet \& Ruiz Liard (2005); } \\
\text { Schunke et al. (2010); Velayos \& Santana (2001); Alves \& } \\
\text { Candido (2009) }\end{array}$ \\
\hline & Eminencia articular & $\begin{array}{l}\text { Okeson (2003); Bumman \& Lotzmann (2000); Ramfjord \& } \\
\text { Ash (1972); Velayos \& Santana (2001); Rubiano (2005) }\end{array}$ \\
\hline & Cóndilo del temporal & $\begin{array}{l}\text { Figún \& G arino (2002); Rubiano (2005); Martinez Ross } \\
\text { (1992) }\end{array}$ \\
\hline & Raíz transversal del cigoma & Figún \& Garino (2002) \\
\hline & Cresta articular & Figún \& Garino (2002) \\
\hline & $\begin{array}{l}\text { Eminentia articulatis } \\
\text { Tuberculum articulare }\end{array}$ & $\begin{array}{l}\text { Langton \& Eggleton (1992) } \\
\text { Velayos \& Santana (2001) }\end{array}$ \\
\hline \multirow[t]{4}{*}{$\begin{array}{l}\text { Fosa Mandibular } \\
\text { (Fossa mandibularis) }\end{array}$} & $\begin{array}{l}\text { Fosa glenoidea } \\
\text { Fossa mandibularis }\end{array}$ & Okeson (2003); Bumman \& Lo tzmann (2000); Rubiano \\
\hline & Cavidad glenoidea & $\begin{array}{l}\text { Sicher \& Dubrul (1991); R amfjord \& Ash (1972); Velayos } \\
\text { \& S antana (2001); Figún \& Garino (2002); Martinez Ross }\end{array}$ \\
\hline & $\begin{array}{l}\text { Fosa articular } \\
\text { Fosa mandibular }\end{array}$ & $\begin{array}{l}\text { Okeson (2003); Figún \& Garino (2002) } \\
\text { Okeson (2003); Sicher \& Dubrul (1991); Ramfjord \& A sh; } \\
\text { Latarjet \& Ruiz Liard (2005); Schunke et al. (2010); } \\
\text { Velayos \& Santana (2001) }\end{array}$ \\
\hline & $\begin{array}{l}\text { Cavidad articular } \\
\text { Cisura petrotimpanica }\end{array}$ & $\begin{array}{l}\text { Schunke et al. (2010) } \\
\text { Okeson (2003) }\end{array}$ \\
\hline \multirow{2}{*}{$\begin{array}{l}\text { Fisura petrotimpanica (Fissura } \\
\text { petrotympanica) }\end{array}$} & Fisura petrotimpanica & $\begin{array}{l}\text { Bumman \& Lotzmann (2000); Sicher \& D ubrul (1991); } \\
\text { Latarjet \& Ruiz Liard (2005); Velayos \& Santana (2001); } \\
\text { Alves \& Candido (2002) }\end{array}$ \\
\hline & $\begin{array}{l}\text { Cisura de Glasser } \\
\text { Cisura petrotimpanica } \\
\text { de Glasser }\end{array}$ & $\begin{array}{l}\text { Latarjet \& Ruiz Liard (2005); Velayos \& Santana (2001) } \\
\text { Figún \& Garino (2002) }\end{array}$ \\
\hline \multirow[t]{2}{*}{$\begin{array}{l}\text { Fisura petroescamosa (Fissura } \\
\text { petrosquamosa) }\end{array}$} & $\begin{array}{l}\text { Cisura petroescamosa } \\
\text { Fisura petroescamosa }\end{array}$ & $\begin{array}{l}\text { Okeson (2003) } \\
\text { Bumman \& Lotzmann (2000); Sicher \& Du brul (1991); } \\
\text { Ramfjord \& Ash (1972); Alves \& Candido (2009) }\end{array}$ \\
\hline & Fisura petrotimpanica & Ramfjord \& Ash (1972) \\
\hline \multirow[t]{2}{*}{$\begin{array}{l}\text { Fisura timpanoescamosa (Fissura } \\
\text { tympanosquamosa) }\end{array}$} & $\begin{array}{l}\text { Cisura escamosotimpanica } \\
\text { Fisura timpanoescamosa }\end{array}$ & $\begin{array}{l}\text { Okeson (2003) } \\
\text { Bumman \& Lotzmann (2000); Sicher \& Du brul (1991); } \\
\text { Alves \& Candido (2009) }\end{array}$ \\
\hline & Fisura & Ramfjord \& Ash (1972) \\
\hline
\end{tabular}

de el proceso cigomático (Processus zygomaticus) hasta la espina del esfenoides (Spina ossis sphenoidalis) (Figún \& Garino; Martinez-Ross).

En la porción medial y anterior de la fosa se inserta un ligamento mencionado por Langton \& Eggleton como ligamento de Tanaka que une al disco con la superficie articular craneal (Sosa). Adicionalmente, en la zona posterior de la fosa, superior a la zona de la fisura petrotimpánica, se inserta la porción superior de la zona bilaminar del disco articular (Langton \& Eggleton).

En el sector posterior de la fosa mandibular se encuentra la fisura timpanoescamosa, que representa el lími- te entre la porción timpánica y escamosa del hueso temporal (Sicher \& Dubrul) (Fig. 2). El techo del tímpano (tegmen tympani) divide a la fisura timpanoescamosa en dos fisuras: una anterior llamada fisura petroescamosa y otra posterior llamada petrotimpanica (Figs. 2 y 3) (Okeson; Sicher \& Dubrul; Bumann \& Lotzmann; Alves \& Candido; Platzer, 2013). Confirmando lo anterior, en un estudio realizado por Anagnostopoulou et al. (2008) en 40 cráneos secos humanos, encontraron en el $92,5 \%$ tres fisuras claramente distinguibles en la fosa mandibular (fisuras timpanoescamosa, petrotimpanica y petroescamosa). Sólo en el 7,5\% restante, la fisura timpanoescamosa no se dividía debidamente en las fisuras petrotimpanica y petroescamosa. 
En los libros de anatomía existen discrepancias al momento de nombrar estas tres fisuras (Tabla I), así como describir su existencia y posición anatómica. Ramfjord \& Ash utilizan los términos fisura petroescamosa y petrotimpanica como sinónimos, y Latarjet \& Ruiz señalan que la fisura petrotimpanica divide la porción escamosa de la timpánica, en lugar de dividir las porciones petrosa y timpánica, como su nombre sugiere. Paulsen \& Waschke (2012) señalan que las fisuras presentes en la fosa se denominan timpanoescamosa, que en la mitad pasa a llamarse petrotimpánica y que por mesial se encuentra la fisura esfenopetrosa, por donde pasa la cuerda del tímpano. Especial confusión provoca el epónimo Cisura de Glasser, ya que algunos autores la utilizan como sinónimo del término fisura petrotimpanica (Latarjet \& Ruiz; Velayos \& Santana; Figún \& Garino; Paulsen \& Waschke) (Tabla I) y otros para referirse a la totalidad de las fisuras presentes en la fosa mandibular (petrotimpanica, timpanoescamosa y petroescamosa) (Martínez Ross).

Importantes estructuras anatómicas pasan por la fisura petrotimpanica entre las cuales destacan las fibras del ligamento esfenomandibular (Ligamentum sphenomandibulare) (cambian de nombre a ligamento anterior del martillo, al pasar por la fisura petrotimpánica ) (Fuentes et al., 2014b), el nervio cuerda del tímpano (Chorda tympani) y la arteria timpánica anterior (Platzer, 2013).

Hay acuerdo en la literatura (Figún \& Garino; Martinez Ross; Alves \& Candido) sobre que las fisuras presentes en la fosa la dividen en dos porciones, siendo sólo la anterior la articular, y que la posterior (no articular) forma la pared anterior del conducto auditivo externo (Martinez Ross). Sin embargo, no existe concordancia sobre el exacto inicio y término de la porción anterior de la fosa. Así, algunos autores señalan que la superficie articular de la fosa se extiende desde la fisura timpanoescamosa (Fig. 2 y 3) (Latarjet \& Ruiz Liard; Alves \& Candido) hasta el margen anterior del tubérculo articular (Latarjet \& Ruiz Liard), mientras que Velayos \& Santana y Sosa indican que la superficie articular se sitúa entre la fisura petrotimpánica y la parte posterior del tubérculo.

En general los autores concuerdan en que la superficie articular de la fosa mandibular está recubierta por fibrocartilago (Schunke et al.; Velayos \& Santana) o tejido fibroso (Martinez Ross; Schunke et al.) y no por cartílago hialino (Schunke et al.), a diferencia de otras articulaciones sinoviales.

Algunos autores mencionan que en la porción medial (Sicher \& Dubrul) y superior de la fosa (Rubiano; Okeson), la pared ósea se adelgaza considerablemente (Sicher \&
Dubrul). Sicher \& Dubrul consideran que la extremada delgadez de la pared ósea de la fosa mandibular es una evidencia clara de que la fosa mandibular, a pesar de contener el margen posterior del cóndilo y del disco, no es una estructura que soporte las cargas funcionales de la ATM, siendo estas cargas soportadas exclusivamente por el cóndilo, disco y tubérculo articular. Figún \& Garino también señalan que la fosa cumple una función pasiva en la ATM y que sólo el cóndilo de la mandíbula y el tubérculo articular son activos.

En cuando a la morfometría de la fosa, Ballesteros Acuña et al. (2011) analizaron 120 ATM en sujetos de más de 20 años, determinando la altura y forma de la fosa mandibular. Para esto trazaron una una línea horizontal que unía el vértice del tubérculo articular con el tubérculo preauricular, y una línea perpendicular a ella, hasta la zona más superior de la fosa, reportando una medida vertical de $6,9 \mathrm{~mm}$. Los resultados indican una reducción significativa de la profundidad de la fosa en pacientes parcialmente dentados o desdentados. Además, las fosas planas predominaron en pacientes edéntulos $(79,9 \%)$ y las profundas en sujetos dentados $(72,3 \%)$, siento esta diferencia significativa. De igual manera, a medida que aumenta la edad se incrementa la posibilidad de encontrar fosas aplanadas. Jasinevicius et al. (2006) tras analizar 134 cráneos en sujetos de más de 21 años, encontraron que la altura de la fosa, medida por la tangente que une el punto superior de la fosa con el plano de Frankfurt (definido por estos autores como la línea que une meato acústico y el foramen infraorbital), presenta diferencias significativas por lado, siendo la fosa derecha más profunda que la izquierda $(6,82 \pm 1,37 \mathrm{~mm}$ al lado derecho y $6,57 \pm 1,29 \mathrm{~mm}$ al izquierdo).

Tubérculo articular (Tuberculum articulare) del hueso temporal. Es una prominencia ósea, muy convexa en sentido antero-posterior (Fig. 2) (Pertes \& Gross, 2005; Sicher \& Dubrul; Figún \& Garino; Alves \& Candido) y levemente cóncava en sentido latero-medial (Sicher \& Dubrul; Figún $\&$ Garino; Alves \& Candido). Por anterior y en él, se inserta el ligamento lateral de la ATM (Langton \& Eggleton; Okeson).

Sicher \& Dubrul señalan que la Terminologia Anatomica genera confusiones con respecto a esta estructura anatómica, ya que la TAI elabora los términos según la función de las estructuras anatómicas, y por lo tanto, debiese existir una clara distinción entre el término tubérculo articular y el término eminencia articular, siendo el primero una estructura no articular sino más bien, una tosca y pequeña saliente (sic) en la superficie lateral del proceso cigomático del hueso temporal, proyectándose debajo de la superficie articular del hueso temporal. Por su parte, la eminencia articular es una estructura ósea transversal, que com- 
prende la estructura articular propiamente dicha, siendo ampliamente recorrida por el cóndilo de la mandíbula y el disco articular. Esta superficie articular se continua con el ápice de la eminencia (sic) (Sicher \& Ducbrul). Rubiano señala que la zona articular termina por delante del ápice del tubérculo, al contrario de Alves \& Candido quienes señalan que es solo la vertiente posterior.

En general, los autores concuerdan en que la superficie articular del tubérculo está recubierta por fibrocartílago (Alves \& Candido; Pertes \& Gross). Oberg et al. midieron las dimensiones de las superficies articulares de los componentes temporales de la ATM, y establecieron una longitud anteroposterior de $19,1 \mathrm{~mm}$ y una mediolateral de 23 $\mathrm{mm}$. En los márgenes de la superficie articular del hueso temporal se inserta la capsula de la ATM (Langton \& Eggleton).

Zabarovic et al. (2000) estudiaron la inclinación del tubérculo articular en 137 cráneos secos de sujetos de más de 18 años, donde la inclinación se define como en ángulo anterior, formado por la pared posterior del tubérculo articular y la línea que uno los puntos craneométricos Porion-
Orbital (línea de Franckfurt); el valor promedio de la inclinación de esta estructura ósea fue de $61,9^{\circ}$, con un rango de $30^{\circ}$ a $94^{\circ}$. No se encontraron diferencias significativas por estado de la dentición (dentado y desdentado) o según el lado, pero si por sexo, siendo la inclinación del tubérculo articular más pronunciada en hombres que en mujeres, mientras que Jasinevicius et al. encontraron diferencias significativas según el lado, siendo la inclinación del tubérculo articular derecho más pronunciada que la del izquierdo.

\section{CONCLUSIÓN}

La descripción anatómica presente en la literatura de las estructuras óseas que componen la ATM es poco exacta; existen importantes diferencias sobre la delimitación del cóndilo mandibular, de las fisuras presentes en la fosa mandibular y de las superficies articulares de la fosa mandibular y tubérculo articular, así como una amplia variabilidad de términos para referirse a una estructura anatómica. Esto dificulta la comunicación entre la comunidad científica y el aprendizaje de los estudiantes de pregrado.

FUENTES, R.; CANTÍN, M.; OTTONE, N. E. \& BUCCHI, C. Characterization of Bone Components of the Temporomandibular Joint. A Literature Review. Int. J. Morphol., 33(4):1569-1576, 2015.

SUMMARY: The temporomandibular joint (TMJ) is a ginglymus-arthrodial bilateral synovial joint, anatomically composed by the mandibular condyle, mandibular fossa and articular tubercle. It is necessary to have a perfect knowledge of the anatomy of the TMJ for a proper understanding of its function. The anatomical description of bony structures of the TMJ present in the scientific literature is not entirely accurate, with several differences on the delimitation of the mandibular condyle, the limits of the articular surfaces, the fissures present in the mandibular fossa. In addition, there is a wide variability of terms for each anatomical structure. A review of textbooks and scientific articles was performed, about the anatomical description of the bony components of the TMJ and terms to refer to them, compared with the terms established by the Anatomical Terminology.

KEY WORDS: Temporomandibular joint; Mandibular condyle; Articular tubercle; Mandibular fossa; Anatomical Terminology.

\section{REFERENCIAS BIBLIOGRÁFICAS}

Alves, N. \& Candido, P. L. Anatomía Aplicada a la Odontología. São Paulo, Santos, 2009.

Anagnostopoulou, S.; Venieratos, D. \& Antonopoulou, M. Temporomandibular joint and correlated fissures: anatomical and clinical consideration. Cranio, 26(2):8895, 2008.

Ballesteros Acuña, L. E.; Ramirez Aristeguieta, L. M. \& Muñoz Mantilla, G. Mandibular fossa depth variations: Relation to age and dental state. Int. J. Morphol., 29(4):1189-94, 2011.
Bumann, A. \& Lotzmann, U. Atlas de Diagnóstico Funcional y Principios Terapeúticos en Odontología. Barcelona, Masson, 2000.

Comité Federal sobre Terminología Anatómica (FCAT). Terminología Anatómica. Terminología Anatómica Internacional. Madrid, Médica Panamericana, 2001.

Figun, M. \& Garino, R. Anatomía Odontológica Funcional y Aplicada. Buenos Aires, El Ateneo, 2002.

Fuentes, R.; Ottone, N.; Cantin, M. \& Bucchi, C. Analysis 
of terms used in scientific literature referred to extracapsular ligaments of the temporomandibular joint. Part II: Ligamentum stylomandibulare and raphe pterymandibulare. Int. J. Morphol., 32(4):1289-95, 2014a.

Fuentes, R.; Bucchi, C. \& Cantin, M. A study of terms used in scientific literature to refer to the extracapsular ligaments of the temporomandibular joint. Part I: Ligamentum sphenomandibulare. Int. J. Morphol., 32(3):981-6, 2014b.

Jasinevicius, T. R.; Pyle, M. A.; Lalumandier, J. A.; Nelson, S.; Kohrs, K. J.; Türp, J. C. \& Sawyer, D. R. Asymmetry of the articular eminence in dentate and partially edentulous populations. Cranio, 24(2):85-94, 2006.

Langton, D. \& Eggleton, T. Functional Anatomy of the Temporomandibular Joint Complex. Tucson, INFORC publications, 1992.

Latarjet, M. \& Ruiz Liard, A. Anatomía Humana. Tomo 2. $2^{\mathrm{a}}$ ed. Buenos Aires, Editorial Médica Panamericana, 2005.

Martínez Ross, E. Disfunción Témporomandibular. Santa Fé de Bogotá, Ediciones Monserrate, 1992.

Oberg, T.; Carlsson, G. E. \& Fajers, C. M. The temporomandibular joint. A morphologic study on a human autopsy material. Acta Odontol. Scand., 29(3):349-84, 1971.

Okeson, J. Tratamiento de Oclusión y Afecciones Temporomandibulares. $5^{\text {a }}$ ed. Madrid, Mosby Inc., 2003.

Paulsen, F. \& Waschke, J. Sobotta. Atlas der Anatomie des Menschen. Kopf, Auge, Ohr. Munich, Elsevier, 2012.

Pertes, R. \& Gross, S. Tratamento Clínico das Disfunçoes Temporomandibulares e da Dor Orofacial. São Paulo, Quintessence, 2005.

Platzer, W. Taschenatlas Anatomie. Band 1: Bewegungsapparat. Stuttgart, Thieme, 2013.

Rubiano, C. M. Tratamiento con Placas y Corrección Oclusal por Tallado Selectivo. Bogotá, Amolca, 2005.

Ramfjord, A. \& Ash, M. Oclusión. 2a ed. Filadelfia, Interamericana, 1972.
Schunke, M.; Schulte, E. \& Schumacher, U. Prometheus. Texto y Atlas de Anatomía. Tomo 3. $2^{\mathrm{a}}$ ed. Buenos Aires, Editorial Médica Panamericana, 2010.

Sicher, H. \& Dubrul, E. Anatomía Oral. $8^{\mathrm{a}}$ ed. Sao Paulo, Artes Médicas, 1991.

Sosa, G. E. Detección Precoz de los Desórdenes Témporomandibulares. Caracas, Amolca, 2006.

Vasconcellos, A. H.; Sousa, E. M. A. \& Cavalcante, M. L. T. M. H. Clasificación de la articulación témporomandibular: aspectos anátomofuncionales. Int. J. Odontostomatol., 1(1):25-8, 2007.

Velayos, J. L. \& Santana H. D. Anatomía de la Cabeza con Enfoque Odontoestomatológico. $3^{\mathrm{a}}$ ed. Madrid, Médica Panamericana, 2001.

Zabarovic, D.; Jerolimov, V.; Carek, V.; Vojvodic, D.; Zabarovic, K. \& Bukovic, D. Jr. The effect of tooth loss on the TM-joint articular eminence inclination. Coll. Antropol., 24 (Suppl. 1):37-42, 2000.

\author{
Dirección para Correspondencia: \\ Dr. Ramón Fuentes Fernández \\ Facultad de Odontología \\ Universidad de La Frontera \\ Manuel Montt 112 \\ Casilla 54-D, Temuco \\ CHILE
}

Email: ramon.fuentes@ufrontera.cl

Recibido : 30-01-2015

Aceptado: 02-09-2015 